\title{
The long-term prognosis and predictors of epilepsy: a retrospective study in 820 patients
}

\author{
Haoran Yang, Jun Zhang, Chenxi Yang, Dan Wu, Xiaoyun Liu, Hongjuan Lu, Wei Zhou and Wei Sun*
}

\begin{abstract}
Background: To investigate the prognosis and predictors for seizure control in epileptic patients in China.

Methods: Eight hundred and twenty patients with epilepsy who visited Xuanwu Hospital from October 2017 to January 2020, were enrolled. The clinical information of the patients was obtained by retrospectively reviewing medical records. Prognostic measures of seizure control included remission, relapse and occurrence of drug resistance. The relationship of prognosis of seizure control with factors such as demographics, clinical characteristics and initial electroencephalography (EEG) features was investigated.
\end{abstract}

Results: A total of 503 (61.3\%) patients experienced a 1-year remission and 330 (49.3\% of 669) had a 2-year remission. Idiopathic type of epilepsy $(P<0.001)$, normal EEG $(P<0.05)$, number of antiepileptic drugs $(P<0.05)$ and seizure frequency of $<1 /$ month $(P<0.001)$ at the first arrival predicted a remission independently. Of the 503 patients who achieved a 1-year remission, 184 (36.6\%) experienced a relapse, due to external reversible causes (58 patients) or with unknown reversible triggers (126 patients). No factors were found to be associated with a relapse $(P>0.05)$. At the end of the study, 322 patients (39.3\%) developed drug resistance. The development of drug resistance was associated with the following factors: symptomatic aetiology of epilepsy, epileptiform abnormality in EEG, number of antiepileptic drugs and seizure frequency of $\geq 1 /$ month at first arrival $(P<0.001)$. For symptomatic epilepsy, patients with meningitis/encephalitis $(P=0.007)$ were more likely to develop drug-resistant epilepsy than these with other causes.

Conclusions: Remission is a common process. The type of epilepsy (idiopathic or symptomatic), EEG features, seizure frequency and treatment history at first arrival are related to both remission and terminal drug resistance. Among various causes of symptomatic epilepsy, meningitis/encephalitis is associated with the worst prognosis of epilepsy.

Keywords: Epilepsy, Prognosis, Remission, Relapse, Drug-resistant epilepsy

\section{Background}

The prognostic factors of epilepsy include seizure control, mortality, as well as social and educational outcomes. Long-term outcomes of seizure control, such as seizure remission [1-3], relapse [4] and drug-resistance [5], can be influenced by many factors, such as the epilepsy type, and causes and frequency of seizure, which are apparent early in the course of diseas e[6]. Understanding the prognostic factors for long-term outcomes

\footnotetext{
*Correspondence: bmusunnyw@163.com
}

Department of Neurology, Xuanwu Hospital Capital Medical University, Beijing 100053, China can provide reference for establishing reasonable treatment plans. For example, for patients who are less likely to obtain a remission, physicians could advice early for nonpharmacological therapies, such as epilepsy surgery or brain stimulation techniques.

Seizure remission is the most common indicator for control of epilepsy. It has been demonstrated that the idiopathic type of epilepsy [6], the low frequency of initial seizure [7, 8], and a rapid response to therapy [9] are predictors for remission, while the symptomatic aetiology of epilepsy is a negative predictor for its prognosis. More detailed predictors for epilepsy control, such as the 
different causes of symptomatic epilepsy, still need to be explored.

In contrast to many studies focusing on remission in a period of time, only a few studies have reported the longterm outcomes after a remission with anti-epilepsy drugs (AEDs) Schiller et al. followed a group of patients who entered a 1-year remission, and found that treatment history is the only factor associated with relapse, while the type of epilepsy is not related to the recurrence of seizures after a remission [4]. Choi et al. followed intractable epilepsy patients and found that 5 out of 20 them who achieved a remission ultimately had an epilepsy relapse. But they failed to found any clinical factors that could predict subsequent seizure relapse. Previous studies have shown that patients who have achieved an AED-induced remission may face a great risk of seizure relapse $[4,10$, 11]. However, the extent and related factors for relapse are still waiting to be investigated.

The development of drug-resistant epilepsy (DRE) is another important outcome of epilepsy. Research on remission did not directly address the issue of development of drug-resistance, as the temporal failure in remission does not necessarily equal to being refractory to treatment. However, DRE has been rarely studied until now, accompanied with different definitions $[3,5,6]$, which makes it difficult to compare results among studies. In 2010, the International League Against Epilepsy (ILAE) proposed a formal consensus definition of DRE, that is, the failure of adequate trials of two tolerated, appropriately chosen and used AED schedules to achieve sustained seizure freedom [12]. This definition provides a standard of what constitutes drug-resistance at a certain time point and allows for selection of a certain epileptic population to study DRE. Moreover, prior research on DRE has mainly focused on pediatric patients $[3,5,13]$, and studies in adult populations were particularly lagging behind.

Previous studies have often focused on a single indicator for prognosis of seizure control, neglecting the combined effect of indicators. Moreover, it is still largely undetermined whether factors related to remission could influence seizure recurrence and drug-resistance. In this study, we performed a retrospective study on outpatients with epilepsy at a single center in China, in order to (i) conclude different outcomes of epilepsy, including 1- or 2-year remission, relapse after an AED-induced remission, and drug-resistance; and (ii) define the clinical factors associated with various outcomes in this population.

\section{Methods}

\section{Patients and data collection}

In this retrospective cohort study, epileptic patients at the outpatient clinic of Xuanwu Hospital, Capital
Medical University, from October 2017 to January 2020, were included. The inclusion criteria were: 1 ) having an established diagnosis of epilepsy; 2) treated by the same physician in our center for more than 1 year, with regular evaluation from once a month to once a year; 3 ) with comprehensive medical records from their first visit to our center to the last visit. Information was mainly obtained from the medical records, consisting of demographics, seizure frequency, age of seizure onset, etiology, the number of AEDs at first visit, family history of epilepsy or febrile convulsions in first-, second- or thirddegree relatives, history of febrile convulsions, electroencephalography (EEG) and magnetic resonance imaging (MRI) results. As most patients had received multiple EEG examinations, only EEG data at the first visit to our center were used. To understand their recent condition, the patients were contacted by telephone at the end of the study. If the patients received epilepsy surgery after inclusion in this study, the observation period ended at the time of the epilepsy surgery.

\section{Study end points \\ Remission criteria}

Remission was defined as the period without occurrence of any type of seizures at any time during the observation time (1 year or longer). If the starting date of remission was not recorded in the medical record, the first clinic visit at which no seizure occurred was regarded as the beginning date of remission.

\section{Relapse criteria}

Relapse was defined as recurrence of any seizure after 1 -year remission. When the specific date of relapse was not noted in the chart, the first visit with seizure recurrence was considered as the date of relapse.

\section{DRE criteria}

DRE was defined as the failure of adequate trials of two tolerated, appropriately chosen and used AED schedules to achieve a sustained seizure remission [14]. The patients were judged at the time of study to see if they met the criteria of DRE.

\section{Classification of epilepsy}

Epilepsy was classified into the idiopathic, symptomatic, and cryptogenic types, according to the guidance of ILAE $[15,16]$. Idiopathic epilepsy, such as juvenile myoclonic epilepsy (JME), is defined as an epilepsy that has age-related onset, specific clinical and EEG characteristics, and a presumed genetic etiology. Symptomatic epilepsy refers to a group with an acquired or genetic cause, including cerebral trauma (head injury and neurosurgery), cerebral tumor, meningitis/encephalitis, stroke, 
cerebral vascular malformations (CMV), mesial temporal sclerosis (MTS), cortical dysplasia (CD), perinatal brain injury, unclear encephalomalacia, neurocutaneous syndromes (tuberous sclerosis and Sturge-Weber syndrome), etc. The determination of causes mainly relies on neuroimaging and the medical history. Cryptogenic epilepsy is considered to have an existing yet unknown cause.

\section{Statistical analysis}

Continuous variables are presented as median, interquartile range (IQR), and range. Categorical variables are presented as counts and percentages. For exploratory purposes, chi-square tests were employed for comparisons of categorical data and the Mann-Whitney test for comparisons of nonparametric continuous data. The variables with significant findings on univariate analysis $(P<0.1)$ would enter the multivariable model. The Cox proportional hazards model was used to investigate the simultaneous effects of prognostic factors to cause remission or relapse. The logistic-regression analysis was used to identify predictors for terminal drug-resistance. $P<0.05$ was considered as statistically significant. Kaplan-Meier survival analysis was first used to estimate the cumulative probability of seizure remission, followed later by considering significant prognostic factors. Statistical analysis was performed using the SPSS software for Windows, version 21.

\section{Results}

Eight hundred and twenty epileptic patients (443, 54.0\% males) were recruited in this study. Of them, 262 (32.0\%) were newly diagnosed patients, whereas the remaining $558(68.0 \%)$ were taking AEDs at the time of first visit to our center. The median age at seizure onset was 15 years (range < 1 year to 77 years; interquartile range [IQR] 10-24). The median duration of epilepsy before the observation was 4 years (IQR 1-11), and the median observation time was 3.6years (IQR 2.4-4.8).

Epilepsy was divided into idiopathic in 125 (15.2\%), symptomatic in 294 (36.0\%), and cryptogenic in 401 patients $(48.8 \%)$. The idiopathic syndromes included JME $(n=68,54.4 \%)$, benign epilepsy with centrotemporal spike (BECTS; $n=43,34.4 \%)$, Jeavons syndrome $(n=4$, $3.2 \%$ ), juvenile absence epilepsy (JAE; $n=4,3.2 \%$ ), childhood absence epilepsy (CAE; $n=4,3.2 \%$ ) and Panayiotopoulos Syndrome $(n=2,1.6 \%)$. The symptomatic epilepsy had etiologies including cerebral trauma $(n=69$, $23.4 \%)$, MTS $(n=65,22.1 \%)$, meningitis/encephalitis $(n=45,15.3 \%)$, unclear encephalomalacia $(n=26,8.8 \%)$, CMV $(n=9,3.1 \%), \mathrm{CD}(n=20,6.8 \%)$, perinatal brain injury $(n=16,5.4 \%)$, neurocutaneous syndromes $(n=3$, $1.2 \%)$, stroke $(n=3,1.2 \%)$, cerebral tumor $(n=2,0.7 \%)$ and others $(n=36,12.2 \%)$.

\section{Remission}

In this population, 503 (61.3\%) patients achieved 1-year remission throughout the entire observation period (Table 1 ). In fact, the majority ( $84.3 \%$ of 503 ) started their first 1-year remission within 1 year after the first visit. The cumulative probability of the first 1-year remission for the overall cohort was $53.1 \%$ (95\% confidence intervals [CI] 51.4-54.8\%), 63.9\% (62.1-65.7\%), 72.6\% (70.4$74.8 \%), 77.9 \%(75.0-80.8 \%)$ at $2,4,6$ and 8 years after the index date, respectively (Fig. 1a). The median time to achieve the first 1-year remission was 1 year (range: 1.0-8.1; IQR: 1.0-1.6years). In the group followed up for at least 2 years, $49.3 \%$ (330/669) of patients entered a remission for at least 2 years. The proportions of patients achieving a 2-year remission at 2, 4, 6 and 8 years after the first arrival was $30.8 \%$ (29.1-32.5\%), 48.6\% (46.6-50.6\%), $58.0 \%$ (55.5-60.5\%) and 64.2\% (61.3-67.1\%) (Fig. 1b).

Univariate analysis revealed significant differences in seizure frequency, number of AEDs at first visit, EEG results, MRI and type of epilepsy between patients achieving a 1-year remission and those who did not (Table 1). Multivariate Cox proportional hazard analysis showed that the seizure frequency, the number of AEDs at the first visit, EEG results and the type of epilepsy were independent predictive factors for seizure recurrence (Table 2). Patients with symptomatic or cryptogenic epilepsy were more likely to achieve a remission than patients with idiopathic epilepsy (symptomatic vs idiopathic: $P<0.001$; cryptogenic vs idiopathic: $P=0.009)$. There was also a significant difference in the probability of remission between symptomatic epilepsy and cryptogenic epilepsy who continued to have seizures $(P<0.001)$. Using survival analysis, longitudinal seizure remission curves were drawn for type of epilepsy (Fig. 2). Patients with high seizure frequency $(<1 /$ month) were 1.48 times more likely to achieve a remission. The patients with normal EEG at the first arrival had higher tendency to achieve a remission than these with epileptiform discharges $(P<0.001)$. In addition, the patients prescribed with AEDs before the first arrival to our center were less likely to achieve a remission than the newly diagnosed epilepsy patients ( 1 AED vs 0 AED, $P=0.037$; $\geq 2$ AEDs vs 0 AED, $P=0.001)$. Moreover, similar associations were found between 2-year remission and seizure frequency, number of AEDs at first arrival, type of epilepsy and EEG results (Table 2).

\section{Relapse after remission}

Of the 503 patients who achieved a remission, 184 (36.6\%) experienced a relapse, including 58 relapses due to external reversible causes and 126 without any known reversible triggers. The external reversible 
Table 1 Demographic and clinical predictors of seizure remission in univariate analysis

\begin{tabular}{|c|c|c|c|c|c|c|}
\hline \multirow[t]{2}{*}{ Variables } & \multicolumn{3}{|l|}{ 1-year remission } & \multicolumn{3}{|l|}{2 - year remission } \\
\hline & Yes $n=503$ & No $n=317$ & $P$-value & Yes $n=330$ & No $n=339$ & $P$-value \\
\hline Sex, male, $n(\%)$ & $280(55.7)$ & $163(51.4)$ & 0.235 & $187(56.3)$ & $177(52.2)$ & 0.281 \\
\hline $\begin{array}{l}\text { Age at seizure onset, years, median } \\
\text { (IQR; range) }\end{array}$ & $15(10-24 ;<1$ to 77$)$ & $16(10-25 ;<1$ to 70$)$ & 0.972 & $15(10-22 ;<1$ to 70$)$ & $16(10-25 ;<1$ to 70$)$ & 0.465 \\
\hline Age at seizure onset, $\leq 12$ years, $n(\%)$ & $168(33.6)$ & $113(35.6)$ & 0.548 & $111(33.6)$ & $116(34.2)$ & 0.874 \\
\hline Seizure frequency, $\geq 1$ /month, $n(\%)$ & $252(50.1)$ & $233(73.5)$ & $<0.001$ & $158(47.9)$ & $244(72.0)$ & $<0.001$ \\
\hline Number of AEDs at first arrival, $n(\%)$ & & & $<0.001$ & & & $<0.001$ \\
\hline 0 & $193(38.4)$ & $69(21.8)$ & & $138(41.8)$ & $84(24.8)$ & \\
\hline 1 & $200(39.8)$ & $123(38.8)$ & & $122(37.0)$ & $137(40.4)$ & \\
\hline$\geq 2$ & $110(21.9)$ & $125(39.4)$ & & $70(21.2)$ & $118(34.8)$ & \\
\hline Family history of epilepsy or FS, $n(\%)$ & $42(8.3)$ & $26(8.2)$ & 0.940 & $28(8.5)$ & $21(6.2)$ & 0.256 \\
\hline History of FS, $n(\%)$ & $51(10.1)$ & $34(10.7)$ & 0.789 & $29(8.8)$ & $38(11.2)$ & 0.297 \\
\hline EEG results, $n(\%)$ & & & $<0.001$ & & & 0.002 \\
\hline Normal & $162(32.2)$ & $51(16.1)$ & & $103(31.2)$ & $66(19.5)$ & \\
\hline Epileptiform abnormality & $266(52.9)$ & $204(64.4)$ & & $176(53.3)$ & $208(61.4)$ & \\
\hline Not done & $75(14.9)$ & $62(19.6)$ & & $51(15.5)$ & $65(19.2)$ & \\
\hline MRI results, $n(\%)$ & & & $<0.001$ & & & $<0.001$ \\
\hline Normal & $293(58.3)$ & $122(38.5)$ & & $201(60.9)$ & $144(42.5)$ & \\
\hline Abnormal & $173(34.4)$ & $170(53.6)$ & & $105(31.8)$ & $169(49.9)$ & \\
\hline Not done & $37(7.4)$ & $25(7.9)$ & & $24(7.3)$ & $26(7.7)$ & \\
\hline Type of epilepsy, n (\%) & & & $<0.001$ & & & $<0.001$ \\
\hline Idiopathic & $101(20.1)$ & $24(7.6)$ & & $71(21.5)$ & $28(8.3)$ & \\
\hline Symptomatic & $135(26.8)$ & $159(50.2)$ & & $86(26.1)$ & $148(43.7)$ & \\
\hline Cryptogenic & $267(53.1)$ & $134(42.3)$ & & $173(52.4)$ & $163(48.1)$ & \\
\hline
\end{tabular}

FS febrile convulsions

triggers included failure to take medicine occasionally (19 patients), discontinuation of AED treatment (16 patients), reduction of the AED dose (4 patients), severe sleep deprivation (7 patients), fever (4 patients), alcohol (3 patients), emotional change (4 patients), and fatigue ( 1 patient). Of the 184 patients who experienced a relapse, 81 had a second 1-year remission, and 58 of them had second remission lasting till the end of the study. The second relapse occurred in 23 patients, and the third relapse in 2 patients.

We next investigated predictive risk factors for seizure relapse after achieving long-term ( $>1$ year) seizure remission. In the univariable analysis, only seizure frequency correlated with relapse $(55.4 \%$ vs $47.0 \%$, $P=0.069$ ), while other variables, including sex, age of seizure onset, seizure frequency, family history of epilepsy or febrile convulsion, history of febrile convulsion, type of epilepsy, EEG and MRI results did not show a significant association with relapse $(P>0.1)$ (Table 3). When seizure frequency was included in the Cox proportional hazards model, there was no significant association between seizure frequency and relapse $(P=0.143)$.

\section{Drug resistance}

At the end of the study, 322 of 820 patients (39.3\%) met the criteria of drug resistance. The prevalence of DRE was $54.4 \%$ (160 of 294 ) in symptomatic epilepsy, $33.7 \%$ (135 of 401) in cryptomatic epilepsy, and $21.6 \%$ (27 of 125 ) in idiopathic epilepsy. In particular, $16.1 \%$ (52 of 322) of patients with DRE had experienced a remission.

In the univariable analysis, the following variables were associated with DRE: seizure frequency and the number of AEDs at first visit, EEG results, MRI and the type of epilepsy (Table 4). Multivariable analysis showed that the seizure frequency and the number of AEDs at first visit, EEG results, and the type of epilepsy remained significantly associated with the likelihood of DRE at the end of the study (Table 5). Patients with symptomatic epilepsy had the highest probability of DRE, followed sequentially by those with cryptomatic epilepsy, and those with idiopathic epilepsy (symptomatic vs idiopathic: $\mathrm{OR}=4.70$ (2.74-8.07), $P<0.001$; cryptomatic vs idiopathic: $\mathrm{OR}=2.26(1.34-3.82), P=0.002$; symptomatic vs cryptogenic: $\mathrm{OR}=2.08(1.44-3.00), P<0.001)$.

In addition, we analyzed the possibility of terminal DRE among patients with symptomatic epilepsy of 

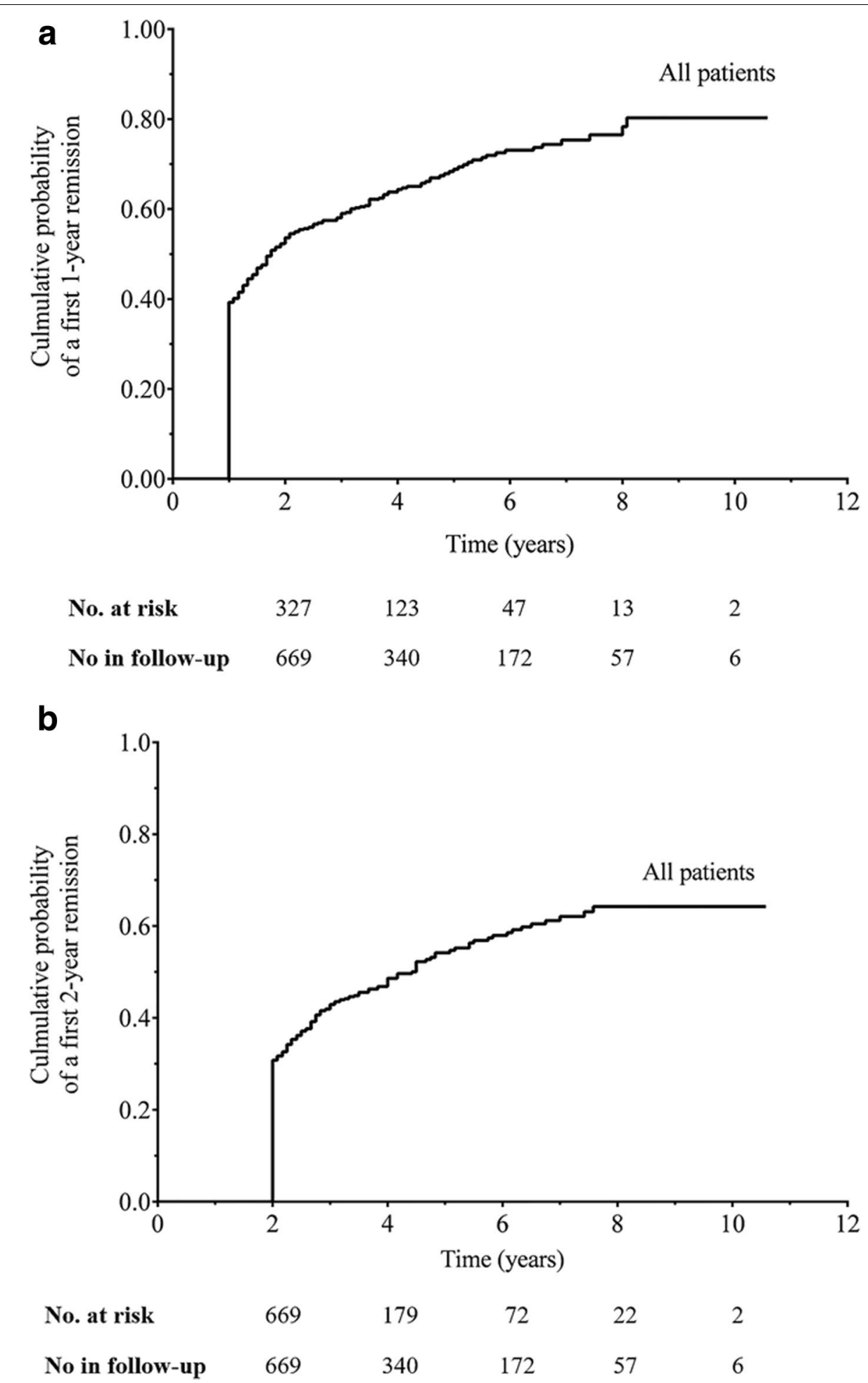

Fig. 1 Kaplan-Meier analyses of time to the first 1-year remission (a), and to the first 2-year remission (b) in the whole cohort 
Table 2 Variables found to correlate with the first 1-year and 2-year remissions in multivariate Cox proportional hazard analysis

\begin{tabular}{|c|c|c|c|c|c|c|}
\hline \multirow[t]{2}{*}{ Variables } & \multicolumn{3}{|c|}{ 1-year remission } & \multicolumn{3}{|c|}{2 - year remission } \\
\hline & HR & $95 \% \mathrm{Cl}$ & $P$-value & HR & $95 \% \mathrm{Cl}$ & $P$-value \\
\hline Seizure frequency & & & $<0.001$ & & & $<0.001$ \\
\hline$\geq 1 /$ month & ref & & & ref & & \\
\hline$<1 /$ month & 1.48 & $1.23-1.77$ & & 1.58 & $1.27-1.98$ & \\
\hline Number of AEDs at first arrival & & & 0.002 & & & 0.008 \\
\hline 0 & ref & & & ref & & \\
\hline 1 & 0.81 & $0.66-0.99$ & 0.037 & 0.75 & $0.58-0.95$ & 0.019 \\
\hline$\geq 2$ & 0.65 & $0.51-0.83$ & $<0.001$ & 0.65 & $0.49-0.87$ & 0.004 \\
\hline EEG results & & & $<0.001$ & & & 0.019 \\
\hline Normal & 1.00 & & & 1.00 & & \\
\hline Abnormal & 0.66 & $0.54-0.82$ & $<0.001$ & 0.73 & $0.57-0.95$ & 0.017 \\
\hline Not available & 0.58 & $0.44-0.77$ & $<0.001$ & 0.65 & $0.46-0.92$ & 0.014 \\
\hline Type of epilepsy & & & $<0.001$ & & & $<0.001$ \\
\hline Idiopathic & ref & & & ref & & \\
\hline Symptomatic & 0.49 & $0.37-0.63$ & $<0.001$ & 0.49 & $0.35-0.67$ & $<0.001$ \\
\hline Cryptogenic & 0.73 & $0.57-0.92$ & 0.009 & 0.67 & $0.50-0.90$ & 0.007 \\
\hline
\end{tabular}

$H R$ hazard ratio, $95 \%$ Cl $95 \%$ confidence intervals, ref reference for odds ratio

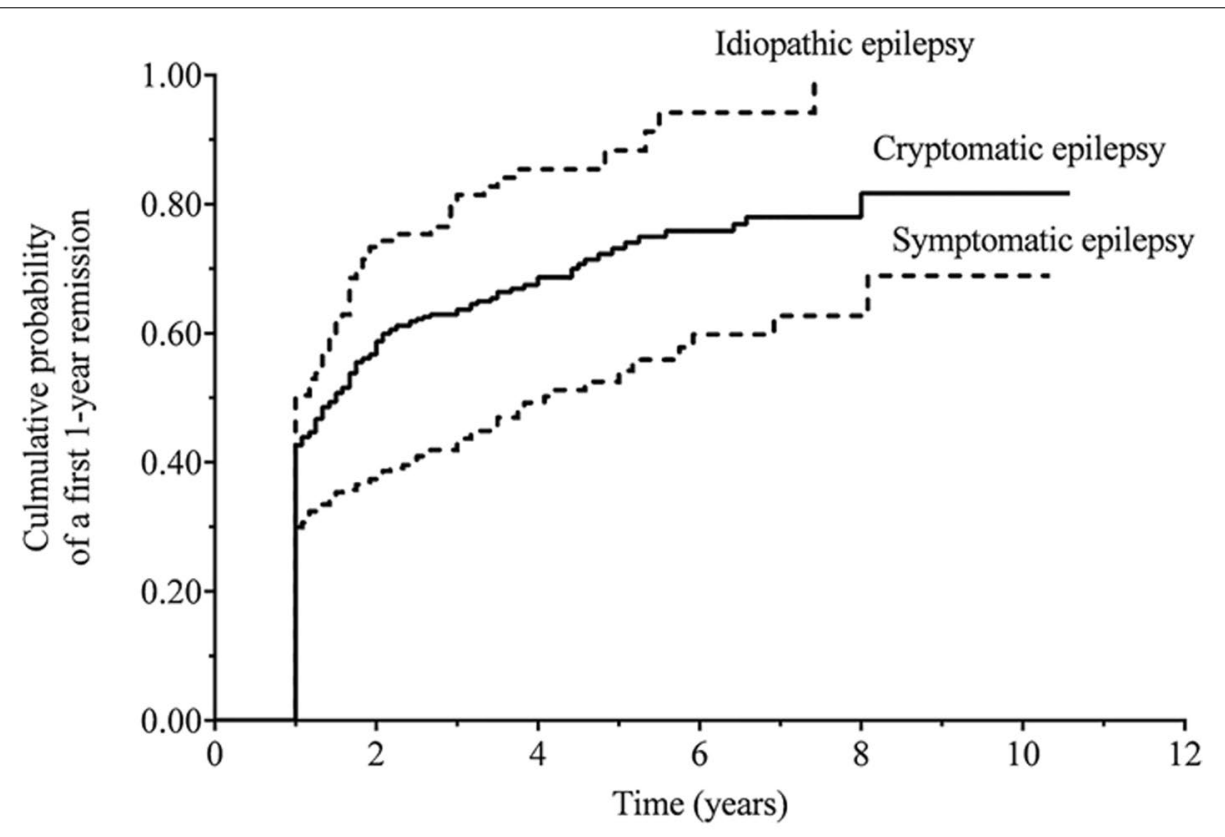

No. at risk/ No in follow-up

$\begin{array}{lccccc}\text { Idiopathic } & 28 / 99 & 10 / 45 & 2 / 19 & 0 / 6 & 0 / 2 \\ \text { Symptomatic } & 151 / 234 & 58 / 115 & 20 / 47 & 7 / 20 & 1 / 2 \\ \text { Cryptomatic } & 148 / 336 & 55 / 180 & 25 / 106 & 6 / 31 & 1 / 2\end{array}$

Fig. 2 Kaplan-Meier analyses of a significant predictive factors for the first 1-year remission: the type of epilepsy 
Table 3 Effect of various factors on seizure relapse after a remission in univariant analysis

\begin{tabular}{|c|c|c|c|}
\hline Variables & Relapse $n=184$ & Not relapse $n=319$ & $P$-value \\
\hline Sex, male, $n(\%)$ & $94(51.1)$ & $186(58.3)$ & 0.116 \\
\hline Age at seizure onset, years, median (IQR; range) & $16(11-25 ; 1-70)$ & $15(10-24 ;<1$ to 77$)$ & 0.298 \\
\hline Age at seizure onset, $\leq 12$ years, $n(\%)$ & $55(29.9)$ & $114(35.7)$ & 0.181 \\
\hline Seizure frequency at first arrival, $\geq 1$ /month, $n$ (\%) & $102(55.4)$ & $150(47.0)$ & 0.069 \\
\hline Family history of epilepsy or FS, $n(\%)$ & $14(7.6)$ & $28(8.8)$ & 0.648 \\
\hline History of FS, $n(\%)$ & $19(10.3)$ & $32(10.0)$ & 0.916 \\
\hline EEG results, $n(\%)$ & & & 0.171 \\
\hline Normal & $50(27.2)$ & $112(35.1)$ & \\
\hline Epileptiform abnormality & $103(56.0)$ & $163(51.1)$ & \\
\hline Not available & $31(16.8)$ & $44(13.8)$ & \\
\hline MRI results & & & 0.773 \\
\hline Normal & $110(59.8)$ & $183(57.7)$ & \\
\hline Abnormal & $63(34.5)$ & $110(34.7)$ & \\
\hline Not available & $11(6.0)$ & $24(7.6)$ & \\
\hline Type of epilepsy, n (\%) & & & 0.113 \\
\hline Idiopathic & $28(15.2)$ & $73(22.9)$ & \\
\hline Symptomatic & $51(27.7)$ & $84(26.3)$ & \\
\hline Cryptogenic & $105(57.1)$ & $162(50.8)$ & \\
\hline
\end{tabular}

FS febrile convulsions

Table 4 Demographic and clinical predictors of drug-resistance in univariate analysis

\begin{tabular}{|c|c|c|c|}
\hline Variables & DRE $n=322$ & Remission $n=388$ & $P$-value \\
\hline Sex, male, n (\%) & $174(54.0)$ & $222(57.2)$ & 0.396 \\
\hline Age at seizure onset, years, median (IQR; range) & $15(10-25 ;<1-70)$ & $15(10-24 ;<1-77)$ & 0.561 \\
\hline Age at seizure onset, $\leq 12$ years, $n(\%)$ & $113(35.1)$ & $140(36.1)$ & 0.784 \\
\hline Seizure frequency at first arrival, $\geq 1 /$ month, $n(\%)$ & $242(75.2)$ & $186(47.9)$ & $<0.001$ \\
\hline Number of AEDs at first arrival, $n(\%)$ & & & $<0.001$ \\
\hline 0 & $49(15.2)$ & $155(39.9)$ & \\
\hline 1 & $134(41.6)$ & $147(37.9)$ & \\
\hline$\geq 2$ & $139(43.2)$ & $86(22.2)$ & \\
\hline Family history of epilepsy or FS, $n(\%)$ & $25(7.8)$ & $34(8.8)$ & 0.631 \\
\hline History of FS, $n(\%)$ & $28(8.7)$ & $40(10.3)$ & 0.467 \\
\hline EEG results at first arrival, $n(\%)$ & & & $<0.001$ \\
\hline Normal & $47(14.6)$ & $135(34.8)$ & \\
\hline Epileptiform abnormality & $217(67.4)$ & $199(51.3)$ & \\
\hline Not available & $58(18.9)$ & $54(13.9)$ & \\
\hline MRI results, $n(\%)$ & & & $<0.001$ \\
\hline Normal & $124(38.5)$ & $227(58.8)$ & \\
\hline Abnormal & $174(54.0)$ & $129(33.4)$ & \\
\hline Not available & $24(7.5)$ & $30(7.8)$ & \\
\hline Type of epilepsy, $n$ (\%) & & & $<0.001$ \\
\hline Idiopathic & $27(8.4)$ & $87(22.4)$ & \\
\hline Symptomatic & $160(49.7)$ & $100(25.8)$ & \\
\hline Cryptogenic & $135(41.9)$ & 201(51.8) & \\
\hline Observation time during study, years, median (IQR; range) & $3.5(2.3-4.7 ; 1.0-10.6)$ & $3.6(2.4-5.0 ; 1.0-12.3)$ & 0.175 \\
\hline
\end{tabular}


Table 5 Variables found to correlate with drug-resistance in the logistic-regression analysis

\begin{tabular}{lll}
\hline Variables & OR (95\% Cl) & $P$-value \\
\hline Seizure frequency, $\geq 1$ /month & $2.62(1.85-3.73)$ & $<0.001$ \\
Number of AEDs at first arrival & & $<0.001$ \\
0 & ref & \\
1 & $2.80(1.82-4.30)$ & $<0.001$ \\
$\geq 2$ & $4.24(2.69-6.67)$ & $<0.001$ \\
EEG results at first arrival & & $<0.001$ \\
$\quad$ Normal & ref & \\
Epileptiform abnormality & $3.13(2.05-4.78)$ & $<0.001$ \\
$\quad$ Not available & $2.75(1.59-4.73)$ & $<0.001$ \\
Type of epilepsy & & $<0.001$ \\
Idiopathic & ref & \\
Symptomatic & $4.70(2.74-8.07)$ & $<0.001$ \\
Cryptogenic & $2.26(1.34-3.82)$ & 0.002 \\
\hline
\end{tabular}

ref reference for odds ratio

different causes, and found that the patients with etiology of meningitis/encephalitis (OR: 2.99, 95\% CI: 1.32 - 6.77, $P=0.007)$ were more likely to develop into DRE than patients with other etiologies (Table 6).

\section{Treatment}

At the end of observation time, 40 subjects $(4.9 \%)$ had been off-medication, 393 (47.9\%) were receiving monotherapy, 308 (37.6\%) were receiving 2 AEDs, 76 (9.3\%) were treated with three drugs, and $3(0.4 \%)$ were taking four. For the patients who were not taking drugs at the end of the study, $80.0 \%$ (32 of 40) of the patients were in a remission, including 21 with idiopathic epilepsy (17 with BECTS, 2 with CAE, 1 with JME, 1 with Panayiotopoulos syndrome), 1 with symptomatic epilepsy and 10 with cryptomatic epilepsy.

Of the patients who were in a remission at the last follow-up, 31 (8.6\%) were taking no drugs, 228 (63.3\%) were taking 1 drug, and 101 (28.1\%) were taking at least 2 drugs. Among the seizure-free patients, $11.5 \%$ of patients with idiopathic epilepsy required 2 or more AEDs (OR: 0.25 , 95\% CI: $0.13-0.51, P<0.001)$ compared with $33.9 \%$ with nonidiopathic epilepsy (41\% with symptomatic epilepsy, 30.3\% with cryptomatic epilepsy).

\section{Discussion}

By retrospectively assessing the outcomes of 820 epilepsy patients, we observed that (i) over half of patients could achieve a remission during the course of epilepsy but with a high rate of subsequent relapse, with some even developing into DRE; (ii) several factors were related to the remission and terminal DRE, including the type of epilepsy (idiopathic or symptomatic), EEG results, seizure frequency and treatment history; and (iii) in the group of symptomatic epilepsy, patients with encephalitis/meningitis etiology had the worst prognosis than those with other etiologies.

\section{Remission}

During the observation time, $61.3 \%$ of patients with epilepsy achieved a 1-year remission of seizures and $49.3 \%$ had a 2-year remission, which clearly showed that the majority of patients could experience a period of seizure freedom. Previous studies $[6,8-10,14]$ have consistently shown that remission is more likely to occur in idiopathic epilepsy and less likely in nonidiopathic epilepsy. However, there are contradictions about the outcomes between cryptomatic epilepsy and symptomatic epilepsy. Some studies have concluded that there is no significant

Table 6 Comparison of patients with drug-resistant epilepsy and with 1-year remission at the end of the study by etiology of symptomatic epilepsy

\begin{tabular}{lllll}
\hline & DRE (\%) $\boldsymbol{n}=\mathbf{1 6 0}$ & Remission (\%) $\boldsymbol{n = 1 0 0}$ & OR (95\% Cl) & P-value \\
\hline Cerebral trauma & $38(23.8)$ & $28(28.0)$ & $0.80(0.45-1.41)$ & $1.42(0.76-2.66)$ \\
Mesial temporal sclerosis & $38(23.8)$ & $18(18.0)$ & $2.99(1.32-6.77)$ & 0.444 \\
Meningitis/encephalitis & $33(20.6)$ & $8(8.0)$ & $0.60(0.25-1.43)$ & 0.273 \\
Unclear encephalomalacia & $11(6.9)$ & $11(11.0)$ & $1.16(0.41-3.23)$ & 0.007 \\
Cortical malformation & $11(6.9)$ & $6(6.0)$ & $0.37(0.12-1.17)$ & 0.245 \\
Perinatal brain injury & $5(3.1)$ & $8(8.0)$ & $1.67(0.30-9.23)$ & 0.781 \\
Cerebral vascular malformations & $4(2.5)$ & $2(2.0)$ & NC & 0.079 \\
Neurocutaneous syndromes & $2(1.3)$ & $1(0)$ & $0.62(0.04-10.07)$ & 0.693 \\
Cerebral tumor & $1(0.6)$ & $2(2.0)$ & NC & 0.262 \\
Stroke & 0 & $16(16.0)$ & $0.70(0.34-1.45)$ & 0.14 \\
Unknown & $17(10.6)$ & $95 \%(1.000$ & 0.334 \\
\hline
\end{tabular}

DRE drug resistant epilepsy, NC not calculated because one of the cells has a zero value, $95 \%$ CI $95 \%$ confidence intervals 
difference in the outcomes between cryptomatic and symptomatic epilepsies [6,17], while others having not $[9,12]$. The present study supports that patients with cryptomatic epilepsy have a better prognosis than these with symptomatic epilepsy. Instead of regarding cryptogenic epilepsy as probably symptomatic, it may be more scientific to consider it as a separate entity with a relatively good prognosis. In addition, we found that patients with AEDs at the first visit had a much lower probability of remission than patients not taking drugs. This is not difficult to explain. Patients who had failed 1 or more AEDs tended to search for better physicians than those who had achieved seizure control. Therefore, this group of patients with a previous treatment had been screened and tended to have a bad prognosis, especially when at least 2 AEDs had failed in them. Moreover, here the abnormal MRI results were associated with remission in univariate analysis, but lost its significance in multivariate analysis. The reason for the above inconsistency may be that the MRI results were highly correlated with the classification of epilepsy.

\section{Relapse after a remission}

We examined the probability of seizure relapse in those who had experienced a remission. We found that 184 of $503(36.6 \%)$ patients relapsed during the observation time after achieving a remission. Interestingly, this degree of relapse was consistent with that seen by Schiller et al. [4], who found that $40 \%$ of patients who achieved a greater than 1-year remission experienced seizure relapse at 5 years after entering seizure remission. Another study reported that in a cohort of 59 patients with refractory epilepsy who entered a 1-year remission, 34 (57.6\%) of them had a relapse [18]. The main difference between this and our cohorts was that Callaghan et al. included only patients with refractory epilepsy, while our study included all patients with epilepsy. All these studies demonstrate that even several years of remission could not guarantee permanent remission. In other words, those who are in a remission may still need many years to get over this disease completely. Physicians must be cautious when discussing prognosis of patients who are in a seizure remission, particularly when planning to decrease dosage or stop medications.

In this study, only a small number of patients experienced a relapse due to medication changes, while a majority (68.5\%) of them experienced a relapse without definite causes, suggesting a fluctuating nature of epilepsy course even with medication. This may be explained by two reasons: the development of drug tolerance after prolonged AED exposure, and the progression of underlying epileptogenesis. In the present cohort, no factors were found to be associated with relapse, including the type of epilepsy and the seizure frequency, which was consistent with previous studies $[4,10,19]$.

\section{Drug resistance}

In our cohort, the prevalence of DRE was 39.3\%, which is similar to $33 \%$ reported by Jose et al. [20]. In both studies, the new definition of ILAE was used. In particular, about one sixth of patients with DRE had experienced a certain period of remission during the observation time, which indicates that drug responsiveness is a dynamic process. Consistent with previous reports, patients with encephalitis/meningitis had the poorest outcomes. TéllezZenteno et al. compared causes in patients with DRE and these without DRE. In their study, DRE was found in $71.4 \%$ of patients due to cerebral infection, which was higher than other causes [20].

\section{Strengths and limitations}

A major strength of our study is the large size of the cohort. Furthermore, all the patients were followed up by the same physician for a long time, with comprehensive medical records, which made it possible to analyze seizure control during the whole observation time. A major limitation is the retrospective cohort study design, which relies on medical records as the major source of information, while other important information may be missed out. Another limitation is the lack of accurate information about treatment efficacy before the first arrival to our center, as some patients had been treated in different hospitals for many years. Therefore, we only examines long-term prognosis from the first arrival to our center.

In conclusion, more than half of patients experienced a remission during the course of epilepsy. However, the remission is not necessarily a persisting process, and the "remitting-relapsing" course may be common. Several factors are related to remission and terminal DRE, such as the type of epilepsy, initial seizure frequency and treatment history. In the group of symptomatic epilepsy, patients with encephalitis/meningitis are significantly more likely to be drug-resistant than those with other causes. These prognostic factors are present early in the course of epilepsy, and can provide reference for making more effective therapies.

\section{Abbreviations \\ AEDs: Anti-epileptic drugs; DRE: Drug-resistant epilepsy; ILAE: International League Against Epilepsy; EEG: Electroencephalography; MRI: Magnetic resonance imaging; JME: Juvenile myoclonic epilepsy; CMV: Cerebral vascular malformations; MTS: Mesial temporal sclerosis; CD: Cortical dysplasia.}

\section{Acknowledgements}

The authors thank all patients and their family members. There were no conflicts of interest to declare. 


\section{Authors' contributions}

Data acquisition and analysis of the manuscript: $\mathrm{YH}$; Data acquisition, analysis and redaction of the manuscript, and also the interpretation of the data: WS: All authors have read and approve of the final version of the manuscript.

\section{Funding}

This work was supported by the National Natural Science Foundation of China (81571267).

\section{Declarations}

\section{Ethics approval and consent to participate}

This was a retrospective analysis of de-identified data collected as part of routine clinical practice. Ethics approval and patient consent was waived.

\section{Consent for publication}

Not applicable.

\section{Competing interests}

Wei Sun is a member of the Editorial Board of Acta Epileptologica. Wei Sun was not involved in the journal's review of, or decisions related to this manuscript.

Received: 18 March 2021 Accepted: 16 September 2021

Published online: 27 October 2021

\section{References}

1. Bonnett L, Smith CT, Smith D, Williamson P, Chadwick D, Marson AG. Prognostic factors for time to treatment failure and time to 12 months of remission for patients with focal epilepsy: post-hoc, subgroup analyses of data from the SANAD trial. Lancet Neurol. 2012;11(4):331-40.

2. Giussani G, Canelli V, Bianchi E, Erba G, Franchi C, Nobili A, et al. Longterm prognosis of epilepsy, prognostic patterns and drug resistance: a population-based study. Eur J Neurol. 2016;23(7):1218-27.

3. Sillanp M. Remission of seizures and predictors of intractability in longterm follow-up. Epilepsia. 1993;34(5):930-6.

4. Schiller Y. Seizure relapse and development of drug resistance following long-term seizure remission. Arch Neurol. 2009;66(10):1233-9.

5. Berg AT, Levy SR, Novotny EJ, Zanchi C, Ferrarese C. Predictors of intractable epilepsy in childhood: a case-control study. Epilepsia. 1996;37(1):24-30.
6. Kwan P, Brodie MJ. Early identification of refractory epilepsy. N Engl J Med. 2000;342(5):314-9.

7. Beghi E, Beretta S, Carone D, et al. Prognostic patterns and predictors in epilepsy: a multicentre study (PRO-LONG). J Neurol Neurosurg Psychiatry. 2019;90(11):320883.

8. Berg AT, Levy SR, Testa FM, Souza DR. Remission of epilepsy after two drug failures in children: a prospective study. Ann Neurol. 2009;65(5):510-9.

9. Sillanpää M, Jalava M, Kaleva O, et al. Long-term prognosis of seizures with onset in childhood. N Engl J Med. 1998;338(24):1715-22.

10. Sillanpää M. Long-term risks following first remission in childhood-onset epilepsy. A population-based study. Epilepsy Behav. 2012;25:145-9.

11. Alsfouk BA, Alsfouk AA, Chen Z, Kwan P, Brodie MJ. Pharmacological outcomes in teenagers with newly diagnosed epilepsy: a 30-year cohort study. Epilepsia. 2019;60(6):1083-90.

12. Wirrell EC, Grossardt BR, Elson L, Nickels KC. A population-based study of long-term outcomes of cryptogenic focal epilepsy in childhood: cryptogenic epilepsy is probably not symptomatic epilepsy. Epilepsia. 2011;52(4):738-45.

13. Ramos-Lizana J, Rodriguez-Lucenilla Ml, Aguilera-López P, Aguirre-Rodríguez J, Cassinello-García E. A study of drug-resistant childhood epilepsy testing the new ILAE criteria. Seizure. 2012;21(4):266-72.

14. Kwan P, Arzimanoglou A, Berg AT, Brodie MJ, Hauser WA, Mathern G, et al. Definition of drug resistant epilepsy: consensus proposal by the ad hoc task force of the ILAE commission on therapeutic strategies. Epilepsia. 2010;51:1069-77.

15. ILAE. Proposal for revised clinical and electroencephalographic classification of epileptic seizures. Epilepsia. 1981;22(4):489-501.

16. ILAE. Proposal for revised classification of epilepsies and epileptic syndromes. Epilepsia. 1989;30(4):389-99.

17. Stephen LJ, Kwan P, Brodie MJ. Does the cause of localisation-related epilepsy influence the response to antiepileptic drug treatment? Epilepsia. 2001:42(3):357-62.

18. Callaghan B, Schlesinger M, Rodemer W, Pollard J, Hesdorffer D, Hauser WA, et al. Remission and relapse in a drug-resistant epilepsy population followed prospectively. Epilepsia. 2011;52(3):619-26.

19. Choi H, Heiman G, Pandis D, Resor SR, Gilliam FG, et al. Seizure remission and relapse in adults with intractable epilepsy: a cohort study. Epilepsia. 2008;49(8):1440-5.

20. Téllez-Zenteno Jose F, Lizbeth H-R, Buckley S, Zahagun R, Rizvi S. A validation of the new definition of drug-resistant epilepsy by the International League Against Epilepsy. Epilepsia. 2014;55(6):829-34.
Ready to submit your research? Choose BMC and benefit from:

- fast, convenient online submission

- thorough peer review by experienced researchers in your field

- rapid publication on acceptance

- support for research data, including large and complex data types

- gold Open Access which fosters wider collaboration and increased citations

- maximum visibility for your research: over $100 \mathrm{M}$ website views per year

At $\mathrm{BMC}$, research is always in progress.

Learn more biomedcentral.com/submissions 